\title{
4D打印液晶弹性体工艺及其性能研究*
}

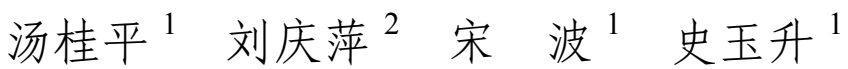 \\ (1. 华中科技大学材料科学与工程学院 武汉 430074; \\ 2. 吉林大学工程仿生教育部重点实验室 长春 130022)
}

\begin{abstract}
摘要: 液晶弹性体(Liquid crystal elastomers, LCEs)因其大且可逆的变形、响应速率快、优异的力学性能、各向异性以及驱动 条件简单灵活等特点, 在智能构件制造领域被认为具有广泛的应用潜力。为了提升液晶弹性体的成形能力, 丰富液晶弹性体 的变形模式及其设计方法, 提出了利用墨水直写打印工艺编程液晶弹性体复杂变形模式的方法。制备了最大变形率达 51\%的 主链型液晶弹性体, 研制适合打印工艺编程的气压挤出高温墨水直写打印设备, 探索了墨水直写打印工艺对液晶弹性体性能 的影响规律。发现打印速率对液晶弹性体的变形率具有显著影响, 随着打印速率的增大, 变形率从 $12 \%$ 逐渐增大到最大变形 率 $51.8 \%$ 后不再变化。此外, 打印温度可使液晶弹性体的变形率在 $38.3 \% \sim 51 \%$ 之间变化, 紫外光照时间可使凝胶分数最高 到 $62.2 \%$, 而打印与紫外光照的间隔时间对材料的变形率没有影响。最后通过打印速率编程液晶弹性体弯曲行为, 实现了液 晶弹性体变形模式和行为的精确控制, 证明了墨水直写打印工艺编程液晶弹性体复杂变形模式方法的可行性。
\end{abstract}

关键词: 液晶弹性体; 墨水直写打印; 工艺编程; 打印速率; 弯曲变形

中图分类号: TG139

\section{Study on 4D Printing of Liquid Crystal Elastomers Process and Properties}

\author{
TANG Guiping $^{1} \quad$ LIU Qingping $^{2} \quad$ SONG Bo $^{1} \quad$ SHI Yusheng ${ }^{1}$ \\ (1. State Key Laboratory of Materials Processing and Die\& Mould Technology, Huazhong University of Science \\ and Technology, Wuhan 430074; \\ 2. Key Laboratory of Bionic Engineering (Ministry of Education), Jilin University, Changchun 130022)
}

\begin{abstract}
Liquid crystal elastomers (LCEs) are considered to have great potential in intelligent component manufacturing due to its large and reversible deformation, fast response, excellent mechanical properties, anisotropy and flexible driving conditions. In order to promote the forming ability of the liquid crystal elastomers and enrich the deformation mode of the liquid crystal elastomers and its design method, a method of programming the complicated deformation mode of the liquid crystal elastomers by the printing process of direct ink writing is proposed. A main-chain liquid crystal elastomer with a maximum deformation rate of $51 \%$ is prepared, and a pressure-extruded high-temperature direct ink writing equipment suitable for printing process programming is developed. The influence of the direct ink writing process on the performance of the liquid crystal elastomers is explored. It was found that the print speed had a significant effect on the deformation rate of the liquid crystal elastomers. With the increase of the print speed, the deformation rate gradually increased from $12 \%$ to the maximum deformation rate of $51.8 \%$ and then no longer changed. Additionally, the deformation rate of the liquid crystal elastomers varies between $38.3 \%$ and $51 \%$ under the control of print temperature, and the UV radiation time can increase gel fraction of the liquid crystal elastomers up to $62.2 \%$. But the interval between printing and UV radiation has no effect on the deformation rate. Finally, the bending behavior of the liquid crystal elastomer was programmed by print speed to achieve precise control of the deformation mode and behavior of the liquid crystal elastomer, which proved the feasibility of the method of programming the complex deformation mode of the liquid crystal elastomers by the direct ink writing printing process.
\end{abstract} Key words: liquid crystal elastomers; direct ink writing; process programming; print speed; bending deformation

* 华中科技大学学术前沿青年团队资助项目(2018QYTD04)。20200401 收到初稿, 20201103 收到修改稿 


\section{0 前言}

随着国家和社会对智能装备的需求越来越 大, 作为智能装备的核心部件, 智能构件受到人 们的广泛关注。智能构件的形状、性能、功能在 外界环境的刺激下能够发生可控的变化, 具有自 感知、自驱动、自适应的特性, 在航空航天、生 物医疗、软体机器人等领域具有极大的应用前 景。智能构件具备形状、性能可控变化等特征, 传统制造方法如铸造、焊接、塑性加工等难以满 足复杂智能构件对结构与材料的双重要求。4D 打印技术是在材料、机械、力学、信息等学科的 高度交叉融合基础上产生的颠覆性制造技术。其 将材料、设计融入制造过程中, 是实现复杂智能 构件制造的有效途径 ${ }^{[1]}$ 。智能材料是 4D 打印智 能构件的重要组成部分, 因此, 高性能智能材料 的制备和成形研究至关重要。

液晶弹性体(Liquid crystal elastomer, LCE) 是 一类由液晶单体通过聚合交联得到的弹性体聚合 物, 同时具有弹性体和液晶相的性质, 具备优异 的力学和光学性能, 如各向异性、刺激响应性、 软弹性和双折射等 ${ }^{[2-5]}$ 。在光、热等外界条件刺激 下, 液晶弹性体能产生相变, 从而发生伸缩变形。 与形状记忆聚合物 (Shape memory polymer, SMP)、电活性聚合物 (Electroactive polymers, EAP)、水凝胶 (Hydrogel) 等智能材料相比, 液晶 弹性体不仅变形程度大且可逆, 而且具有各向异 性、优异的力学性能、多样的驱动条件等特点 ${ }^{[6,7]}$, 因此其在驱动器 ${ }^{[8-10]}$ 、软体机器人 ${ }^{[11-13]}$ 、医疗器 械 ${ }^{[14-16]}$ 等领域具有广泛的应用潜力。液晶弹性体 的伸缩变形功能基于液晶基元定向, 较高取向有 序程度的液晶弹性体有较大的变形率。通常的液

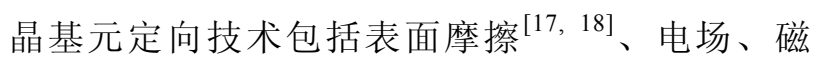
场 $^{[19-22]}$ 、机械拉伸 ${ }^{[23-27]}$ 、光取向方法 ${ }^{[7,28,29]}$ 等, 然而, 这些方法能定向的材料厚度均受到限制, 随着液晶弹性体厚度的增加, 其基元取向有序性 逐渐变差。或者仅限于简单的响应模式, 严重限制 了液晶弹性体的应用。智能构件通常要求响应的精 准可控性, 因此其结构往往具有复杂化、精细化的 特点, 而传统的液晶弹性体制备技术大多是通过模 具完成的, 无法满足智能构件成形的需求。

墨水直写打印技术(Direct ink writing, DIW)因 为复杂形状和结构的成形能力、装备简单、操作方 便、能耗低、可打印材料范围广等特点被广泛应用
于生物材料、高分子和各种复合材料的成形。由于 DIW 技术在打印液晶弹性体的同时，可实现液晶基 元定向, 使其在液晶弹性体器件制备方面显示出突 出的优势。美国德克萨斯大学 WARE 等 ${ }^{[30]}$ 和西班牙 CARLOS 等 ${ }^{[31]}$ 几乎同时利用墨水直写打印制备出 了液晶弹性体, 通过打印路径对材料的取向进行控 制，从而实现了多种变形模式。随后，美国哈佛大 学 LEWIS 等 ${ }^{[32]}$ 用类似的方法制备了液晶弹性体, 并且展示了液晶弹性体平面一三维以及三维一三维 结构的可逆变形能力和优异的驱动性能。瑞士 ANDRÉ 等 ${ }^{[33]}$ 证实了液晶分子的取向可以使材料的 强度、刚度和韧性提高一个数量级, 可以与性能最 好的轻质复合材料相媲美。他们发现液晶分子的取 向性受到打印工艺参数的影响。此外, 还证实了沿 应力方向的打印路径相对其他取向具有更高的材料 强度和刚度。综上所述, 液晶弹性体的变形设计相 关研究相对较少, 且现阶段大多数研究仅仅利用打

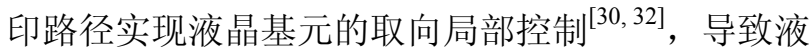
晶弹性体器件变形模式相对较少, 变形设计能力受 到较大限制。

针对以上问题，本文研发了一种可实现在线工 艺编程的墨水直写打印(Direct ink writing, DIW)装 置, 提出了一种新的 4D 打印液晶弹性体复杂变形 设计方法。研究了 DIW 打印工艺对液晶弹性体微观 结构及性能的影响规律, 建立成形工艺-材料性能的 关系，并利用打印速率编程设计和制备了具有可控 复杂变形模式的液晶弹性体器件, 丰富了液晶弹性 体变形模式并拓宽了其应用。

\section{1 试验材料与方法}

\section{1 试验材料}

试验中制备液晶弹性体的原始材料包括三种:

(1)液晶单体 $\mathrm{RM} 82\left(\mathrm{C}_{39} \mathrm{H}_{44} \mathrm{O}_{10}\right)$, 纯度 $96 \%$, 中国予 善化工提供; (2)正丁胺 $\left(\mathrm{C}_{4} \mathrm{H}_{11} \mathrm{~N}\right)$, 纯度 $99 \%$, 美国 Sigma-Aldrich 公司提供; (3)光引发剂 Irgacure369, 纯度 $99.9 \%$, 意大利 BASF 公司提供。在交联密度 的测定过程中使用的试剂有二氯甲烷 $\left(\mathrm{CH}_{2} \mathrm{Cl}_{2}\right)$, 分 析纯, 国药集团化学试剂有限公司提供。以上材料 购买后未经进一步处理直接使用。

\section{$1.2 \mathrm{LCE}$ 的制备}

利用胺烯迈克尔加成反应原理实现增长碳链的 目的。由加成反应得到 LCE 齐聚物后, 再通过紫外 光照使其发生光聚合反应得到液晶弹性体交联网 络。LCE 制备路线如图 1 所示 ${ }^{[32]}$ 。 


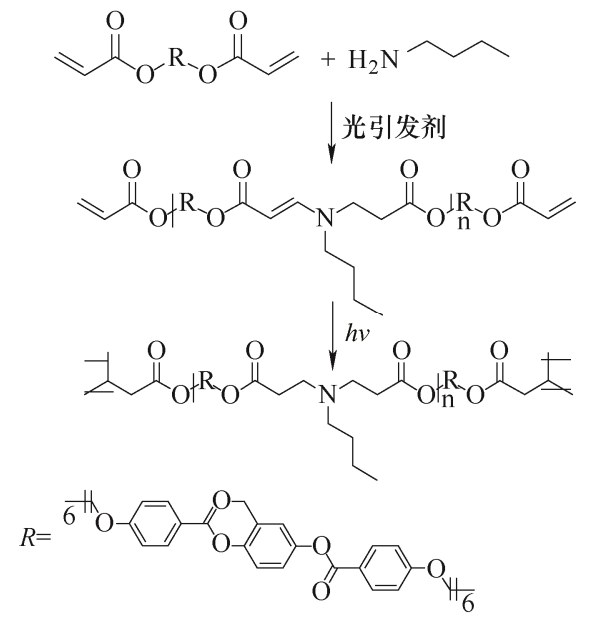

图 1 液晶弹性体合成路线图

液晶弹性体齐聚物的合成工艺如下: 液晶单体 RM82 与扩链剂正丁胺以摩尔比 1:1 混合加入到圆 底烧瓶中, 然后加入前述两者总质量 $1.5 \%$ 的光引发 剂 Irgacure369, 接着在 $85 \sim 110{ }^{\circ} \mathrm{C}$ 下加热, 并持续 搅拌 $15 \sim 18 \mathrm{~h}$ 。

\section{$1.34 \mathrm{D}$ 打印设备和工艺}

液晶弹性体 4D 打印使用的是自主研制的墨水 直写打印设备, 如图 2 所示。具体技术指标见表 1 。 设备主要包括三维移动平台、温度控制、气压挤出 和软件控制等几个工作模块。打印基板可在 $X$ 轴和 $Y$ 轴两个方向移动。 $Z$ 轴导轨和电动机也固定在设 备上, 而整个打印头, 包括料筒、针头和加热组件 可以在 $Z$ 轴导轨上下移动。

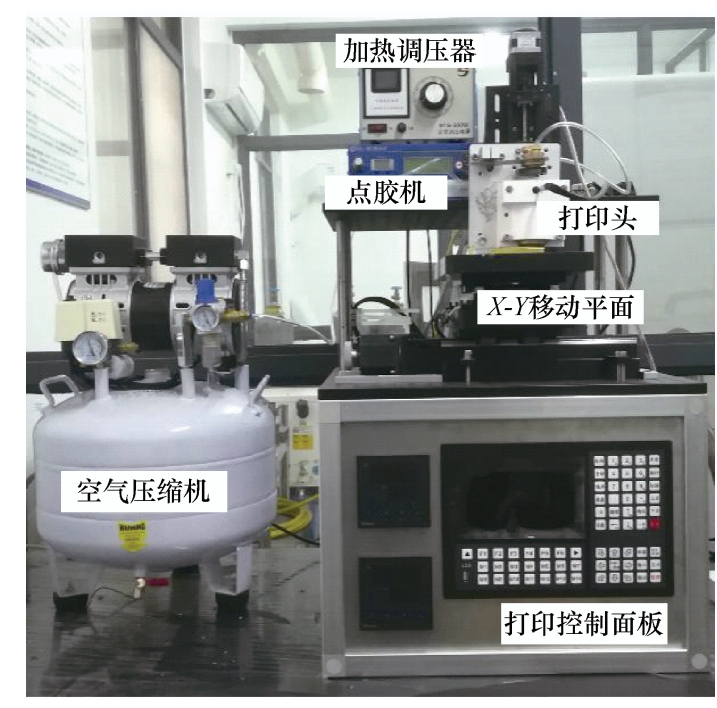

图 2 自主研制的墨水直写打印设备实物图

液晶弹性体齐聚物在常温下为黏度很高的黏 弹性体, 随着温度的上升, 材料逐渐从固态向液 态转变, 黏度大大降低。由于材料的黏度对于墨 水直写打印工艺来说是一种非常重要的参数, 直
接决定了材料的打印成形能否成功, 因此, 通过 温度对黏度进行调控成为改善 LCE 打印性能最 重要的手段之一。

表 1 墨水直写打印装备实验平台性能指标

\begin{tabular}{cc}
\hline 参数 & 内容 \\
\hline 加热温度 $/{ }^{\circ} \mathrm{C}$ & 室温 $\sim 150$ \\
气压压力 $/ \mathrm{MPa}$ & $0.07 \sim 0.8$ \\
成形精度 & - \\
控制软件 & 多普康微数控 $\mathrm{M} 2 \mathrm{P}$ 高配版 \\
成形材料 & 液晶弹性体、高分子、陶瓷浆料等 \\
成形尺寸 & $200 \mathrm{~mm} \times 200 \mathrm{~mm} \times 100 \mathrm{~mm}$ \\
\hline
\end{tabular}

设备的加热元件内部结构如图 3 所示。加热线 圈嵌在一个圆柱形的铜块上, 而铜块直接包覆在料 筒外表面, 可以同时为筒身和针头部位精准加热, 避免材料在针头位置温度急剧降低而黏度快速增加 堵塞针头。同时，铜的高热导性可以保证加热的均 匀性。铜块壁上有一个盲孔, 用于固定热电偶, 设 置在这个位置既是为了避免影响料筒的安装和卸 载, 也为了使测量温度尽可能接近打印材料的实际 温度。铜块安装在特氟龙保温外壳里面, 特氟龙材 料具有良好的隔热作用，能够使得针筒内保持稳定 的温度场。

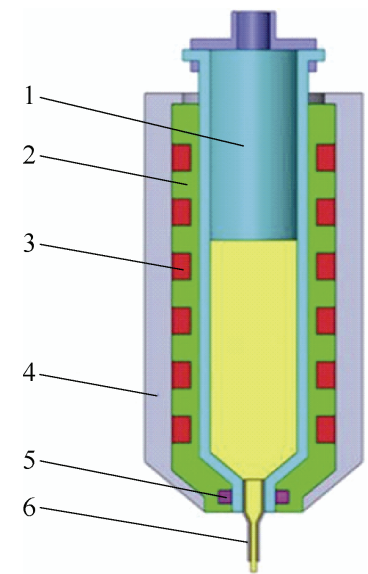

图 3 加热元件内部结构示意图

1. 料筒 2 . 导热铜块 3 . 加热线圈 4. 特氟龙保温外壳 5. 热电偶 6 . 挤出针头

墨水直写打印工艺是一种把黏弹性材料装进料 筒中，然后通过压力把材料从料筒经细小针头挤出 的过程。为了保证打印质量, 对材料挤出过程通常 有以下要求: (1) 稳定性要求, 即出料均匀, 不断线; (2) 挤出开始则开始出料, 挤出停止则停止出料, 即 挤出命令与实际挤出结果间的时延越短越好; (3) 实 际挤出量与设计值一致。为了满足这些要求，除了 
材料要满足某些性能标准外, 对设备也提出了相应 的要求, 而挤出模块的设计就是重点之一。设计的 气压式挤出模块如图 4 所示。

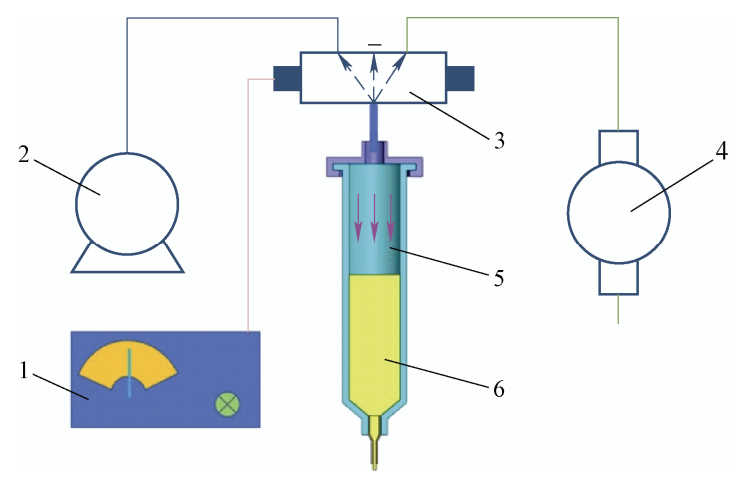

图 4 气压挤出模块

1. 气压控制器 2. 压缩空气源 3. 气动阀

4. 真空㫤 5. 针筒 6. 打印材料

压缩气体由空压机产生并输送至压力控制器, 压力控制器可以设置输出的气压大小, 从而调节挤 出压力值改变材料挤出速度。设备启动打印时, 压 力控制器控制气动阀输出气体通至料筒, 实现材料 的顺利挤出。挤出停止时, 控制器控制气动阀换向, 将针筒与真空原接通使针筒产生负压, 将材料回抽 以防止溢出, 回抽量可以通过调节真空大小和接通 时间控制。回抽动作完成后, 控制器控制气动阀回 到封闭的中间位置, 使针筒内压力封闭, 直到重新 开始打印时再切换到左边的位置与压缩空气联通。

打印过程如下: 先把待打印材料装进料筒, 然 后把料筒装入打印头。调好打印工艺参数, 开启设 备进行预热, 预热方法是把材料加热到打印温度后 再保温至少 $20 \mathrm{~min}$ 。预热结束后开始打印, 打印完 后进行紫外光照固化, 紫外光功率密度为 $0.8 \mathrm{~mW} / \mathrm{cm}^{2}$ 。打印时工艺参数设置为打印速率 $120 \mathrm{~mm} / \mathrm{min}$, 打印温度 $80{ }^{\circ} \mathrm{C}$, 紫外光照时间 $20 \mathrm{~min}$, 挤出压力为 $180 \mathrm{kPa}$, 针头直径为 $0.41 \mathrm{~mm}$, 打印与紫外光照之间无间隔时间。并设置了试验参 数组, 见表 2 , 其中间隔时间为打印结束与紫外光 照之间的间隔时间。

\section{表 2 液晶弹性体打印工艺试验参数设置}

\begin{tabular}{cc}
\hline 工艺参数 & 水平 \\
\hline 打印速率 $/(\mathrm{mm} / \mathrm{min})$ & $10,30,50,70,90,110$ \\
打印温度 $/{ }^{\circ} \mathrm{C}$ & $75,80,85,90,95,100,105,110$ \\
紫外光照时间 $/ \mathrm{min}$ & $0.5,1,2,3,4,5,6,7,8$ \\
间隔时间 $/ \mathrm{min}$ & $0,3,5,10,20,40,70$, \\
\hline
\end{tabular}

\section{4 表征方法}

LCE 齐聚物的相变温度由差示扫描量热法
(Differential scanning calorimetry, DSC)在氮气氛围 下测定，所使用的 DSC 设备为 Diamond DSC(PerkinElmer Instruments, USA)。测试前先把材 料加热到 $200{ }^{\circ} \mathrm{C}$ 以消除材料热历史, 然后冷却 到 $-50{ }^{\circ} \mathrm{C}$, 接着加热到 $200{ }^{\circ} \mathrm{C}$, 升降温速率均为 $10{ }^{\circ} \mathrm{C} / \mathrm{min}$ 。

材料流变性能测试在 Discovery HR-3 Hybrid 流 变仪(TA Instruments, New Castle, DE)上进行。测试 前保持在固定温度 $5 \mathrm{~min}$ 以达到热力学稳定状态, 分别测试在 $50{ }^{\circ} \mathrm{C} 、 80{ }^{\circ} \mathrm{C}$ 和 $110{ }^{\circ} \mathrm{C}$ 时剪切速率在 $0.01 \sim 100 \mathrm{~s}^{-1}$ 范围内变化所对应的黏度。此外, 保持 振荡频率为 $1 \mathrm{~Hz}$, 在 $50{ }^{\circ} \mathrm{C} 、 80{ }^{\circ} \mathrm{C}$ 和 $110{ }^{\circ} \mathrm{C}$ 这三 个不同温度下测定材料在所受剪切应力从 $0.1 \mathrm{~Pa}$ 增 加至 $1000 \mathrm{~Pa}$ 时的动态模量。

液晶弹性体的液晶基元取向有序程度通过二维 广角 X 射线散射(2D Wide Angle X-ray Scattering, 2D-WAXS) 定量测定, 测试所使用的设备是组合化 学 X 射线衍射仪(D8 DISCOVER GDDDS, Bruker)。 测试采用透射模式, 样品厚度约 $0.3 \mathrm{~mm}$ 。有序程度 $S$ 通过以下公式计算

$$
\begin{aligned}
& S=1-N^{-1} \frac{3}{2} \int_{0}^{\frac{\pi}{2}} I(\theta)\left[\sin ^{2} \theta+\right. \\
& \left.\left(\sin \theta \cos ^{2} \theta\right) \lg \frac{1+\sin \theta}{\cos \theta}\right] \mathrm{d} \theta
\end{aligned}
$$

式中, $N=\int_{0}^{\frac{\pi}{2}} I(\theta) \mathrm{d} \theta^{[34]}$ 。

凝胶分数定义为液晶弹性体试样浸入二氯甲烷 中一周前后的干重比, 表征液晶弹性体的交联密度。 用于测试的液晶弹性体试样每一组 3 个试样, 分别 称量其重量, 记为 $W_{i}$ 。把试样完全浸没在液体中一 个星期后取出, 放入 $80{ }^{\circ} \mathrm{C}$ 的烘箱中干燥, 持续 $24 \mathrm{~h}$, 然后称量每个试样的重量, 记为 $W_{f}$, 则液晶弹性体 的凝胶分数 GF 由以下公式计算得出

$$
\mathrm{GF}=\frac{W_{f}}{W_{i}} \times 100 \%
$$

变形率定义为在外界环境刺激下试样变形前后 的长度变化量与初始长度的比值, 表征液晶弹性体 的变形性能。测试时先把试样放置在加热台上, 温 度设置为 $150{ }^{\circ} \mathrm{C}$, 然后开启热台电源进行加热, 直 至温度在 $150{ }^{\circ} \mathrm{C}$ 保持 $5 \mathrm{~min}$, 且液晶弹性体长度不 再发生明显变化。利用相机拍摄整个加热过程。通 过软件测量试样变形前后的长度, 分别记为 $L_{i}$ 和 $L_{f}$, 那么, 变形率 $\varepsilon$ 由以下公式求出

$$
\varepsilon=\frac{L_{i}-L_{f}}{L_{i}} \times 100 \%
$$

弯曲曲率定义为试样弯曲变形后曲率圆半径的 
倒数, 表征液晶弹性体的弯曲变形性能。具体测定 方法是用数码相机拍摄液晶弹性体变形结束后的形 状和尺寸, 然后利用软件测量图片中材料弯曲的圆 半径 $r$, 弯曲圆半径 $r$ 的倒数就是弯曲的曲率。

\section{2 实验结果分析}

\subsection{LCE 齐聚物的性能}

图 5 是 $\mathrm{LCE}$ 齐聚物的 DSC 曲线, 可以发现, 材料存在两个相变点, $-15{ }^{\circ} \mathrm{C}$ 为玻璃化温度 $T_{\mathrm{g}}$, 另 一个在 $98{ }^{\circ} \mathrm{C}$ 附近, 为向列相-各向同性相转变温度 $T_{\mathrm{NI}}$ 。当材料处于 $T_{\mathrm{g}}$ 与 $T_{\mathrm{NI}}$ 之间时为液晶相, 当温度 高于 $T_{\mathrm{NI}}$ 时材料转变为各向同性态。材料需要在液 晶态下进行液晶基元的定向, 因此 DIW 打印温度需 设定在 $T_{\mathrm{g}}$ 和 $T_{\mathrm{NI}}$ 之间。

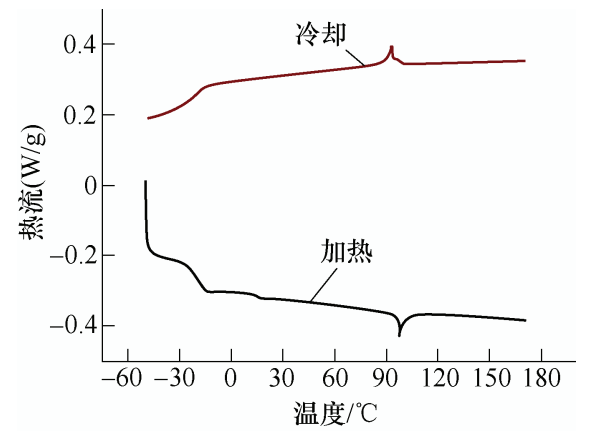

图 $5 \mathrm{LCE}$ 齐聚物的 DSC 曲线

图 6 所示为材料的动态模量与剪切应力之间的 关系曲线, 从图 6 中可以看出, 在这三种温度下材 料的储能模量均小于耗散模量, 表明该温度下液晶 弹性体齐聚物呈流体状, 而非凝胶态或者固态。从 图 7 所示的黏度-剪切速率曲线可以发现, 当剪切速 率约大于 $50 \mathrm{~s}^{-1}$ 时, 材料的黏度明显降低, 即剪切 变稀现象。

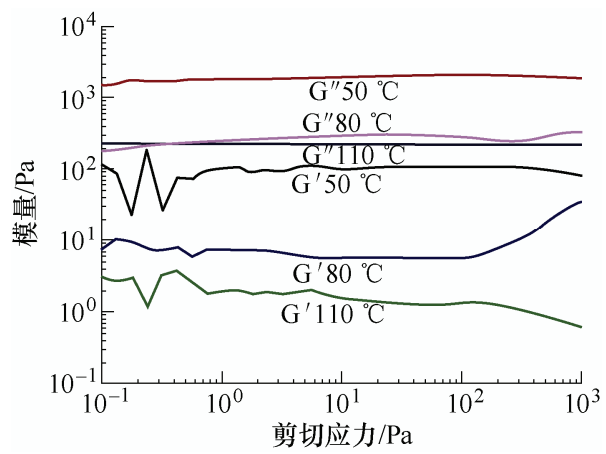

图 $6 \mathrm{LCE}$ 齐聚物的动态模量曲线

剪切变稀性能对于直写工艺非常重要, 因为当 材料从针管中挤出时, 材料受到剪切力, 要使材料 能够顺利从孔径达微米级的针管中挤出, 材料的黏

度必须足够低，而当材料挤出到基板或者先打印出 的材料基体上时必须能够保持形状，否则打印件难 以达到目标件的尺寸精度，因此，此时打印材料应 该有足够高的黏度。此外, 由于液晶弹性体材料的 基元取向是通过挤出时产生的剪切力实现, 因此, 合适的黏度对于液晶弹性体的取向排列也具有重要 意义。

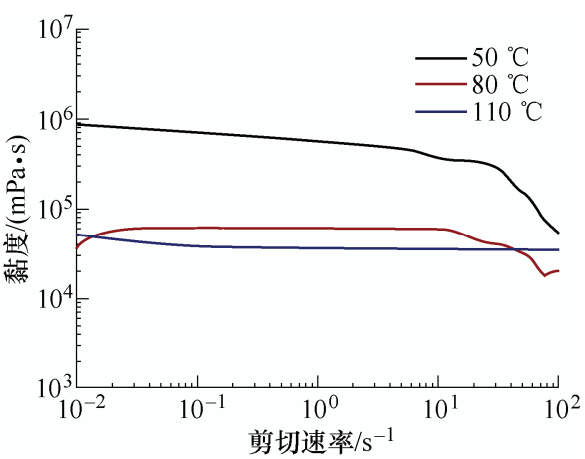

图 $7 \mathrm{LCE}$ 齐聚物的黏度曲线

\subsection{DIW 打印工艺对 LCE 性能的影响}

墨水直写工艺包括两个速率，一个是打印材料 从针头中挤出的速率 $C_{1}$, 挤出速率可以通过压缩空 气的压力大小来调节; 另一个是打印针头的移动速 率 $C_{2}$, 可通过电机控制, 这里所说的打印速率是指 针头的移动速率 $C_{2}$ 。图 8 是液晶弹性体取向有序程 度以及变形率与打印速率之间的关系图, 比较图 $8 \mathrm{a}$ 和 $8 \mathrm{~b}$ 可以发现，对于高打印速率下制备的液晶弹性 体，其图形更为收敛，表明液晶弹性体有序度更高。 从图 8c 中可以看到, 随着打印速率的增大, 变形程 度从 $12 \%$ 逐渐增大, 并趋近于一个稳定值, 最高为 $51.8 \%$ 。而且随着打印速率的增大, 取向有序度跟变

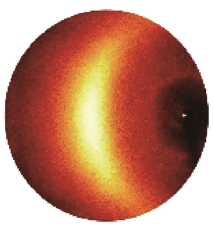

(a) 打印速率为 $10 \mathrm{~mm} / \mathrm{min}$ 时的 $2 \mathrm{D}-\mathrm{WAXS}$ 图

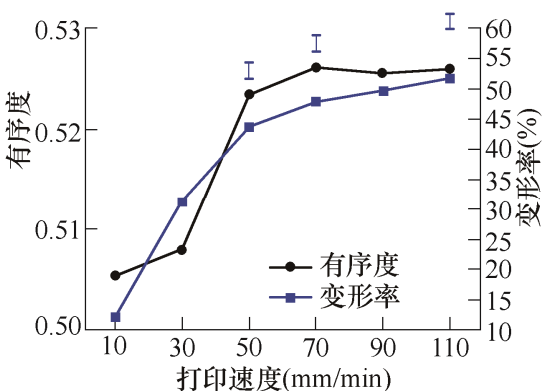

(c) 有序度和液晶弹性体变形率与 打印速率之间的关系曲线

图 8 液晶弹性体的取向有序度以及变形率 
形率有较一致的增长趋势。JENNIFER 等 ${ }^{[32]}$ 的研究 也表明, 随着打印速率的增大, 液晶基元有序程度 也随之增大。当挤出速率 $C_{1}$ 一定时, 单位时间内挤 出的材料量不变, 增大针头的移动速率 $C_{2}$, 即打印 速率, 材料在沉积到基板的过程中所受到的拉力就 会变大, 其被拉长的幅度变大, 从而影响液晶弹性 体的基元有序程度和打印的线条尺寸。

如图 9 所示, 尺寸变化趋势与液晶弹性体变形 率的变化趋势正好相反, 但两者变化速率基本保持 一致。液晶弹性体的这一特性对材料器件的结构和 性能调控具有非常重要的意义。因为在实际应用场 景中, 除了需要较为复杂的结构, 往往要求器件能 够实现复杂的形状变换, 而采用传统成形方法很难 得到复杂的结构, 且复杂的形状变换就需要复杂的 多畴液晶弹性体, 从而就需要很复杂的工艺, 这极 大地限制了液晶弹性体的实际应用。但是, 如果采 用直写工艺来成形, 就可以在得到复杂形状的同时, 还利用 DIW 打印工艺对材料结构和性能进行调控, 实现器件复杂的形状变换。

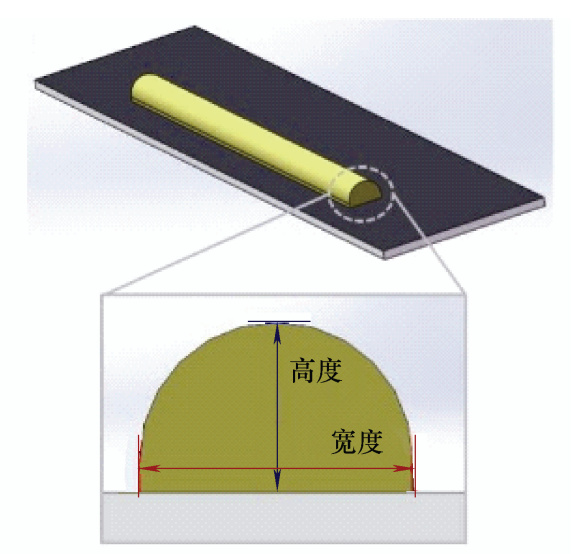

(a) DIW打印液晶弹性体单一线条尺寸测量示意图

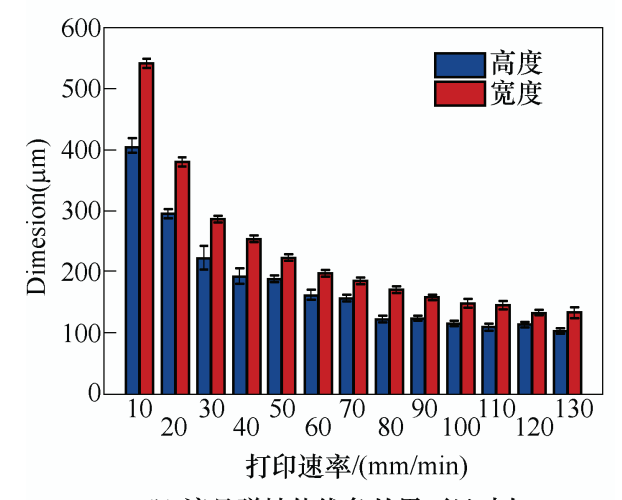

(b) 液晶弹性体线条的界面尺寸与 打印速率之间的关系曲线

图 9 液晶弹性体打印线条的截面尺寸

温度对 DIW 直写工艺影响较大。因为温度不仅 会通过影响材料的流变性影响打印效果, 而且会影 响液晶基元的流动行为, 进而改变其取向的有序度。
为了研究打印温度对材料取向的影响规律, 在不同 温度下打印了 $30 \mathrm{~mm} \times 6.5 \mathrm{~mm} \times 0.3 \mathrm{~mm}$ 的长条, 然后 通过测量其在液晶相和各向同性相之间的尺寸变化 来计算材料的取向有序程度。当温度低于 $75{ }^{\circ} \mathrm{C}$ 时, 出现材料无法挤出或者不正常出料的情况, 因此打 印温度下限设定为 $75{ }^{\circ} \mathrm{C}$ 。由前面的 DSC 曲线可知, 本实验合成的液晶弹性体 $T_{\mathrm{NI}}$ 约为 $100{ }^{\circ} \mathrm{C}$, 故把打 印温度上限设为 $110{ }^{\circ} \mathrm{C}$ 。图 10 为液晶弹性体变形 率与打印温度的关系图。

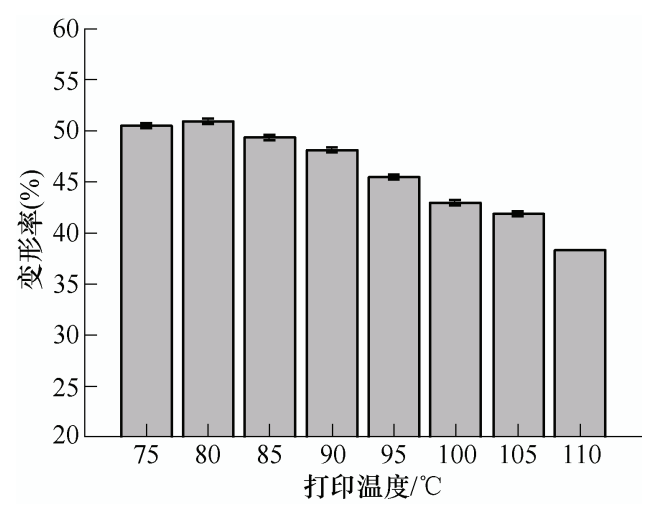

图 10 液晶弹性体变形率与打印温度的关系图

从图 10 中可以看到, 随着温度的上升, 变形率 逐渐下降, $75 \sim 110{ }^{\circ} \mathrm{C}$ 的打印温度可调控液晶弹性 体的变化率在 38.3\% 51\%之间。有两方面的原因, 一方面当温度较低时, 液晶弹性体油墨在挤出过程 以及沉积到基板过程中, 受到的拉力和剪切力较大, 使液晶基元因受力而得到沿挤出方向的取向。另一 方面, 当材料温度较高时, 打印以后材料尚有余热, 使得取向有序的液晶基元向无序转变。

液晶弹性体齐聚物在紫外光辐射下发生交联反 应得到液晶弹性体，从而把液晶基元的取向有序性 固定下来, 使液晶弹性体可在外界刺激下发生可逆 响应。图 11 所示为材料的凝胶分数与紫外光辐射时 间的关系。当光照时间少于 $30 \mathrm{~s}$ 时, 材料交联程度 过低, 浸入二氯甲烷以后将很快溶解, 因此, 收集 到的数据从 $30 \mathrm{~s}$ 开始。从图 11 中可以发现, 当辐 射时间为 $30 \mathrm{~s}$ 时凝胶分数为 $40 \%$, 直到 $4 \mathrm{~min}$ 时才 达到 $60 \%$, 继续增加光照时间材料的凝胶分数基本 保持在这个值不变, 表明在该组分和紫外光照密度 下, $4 \mathrm{~min}$ 的光照时间已经使材料达到了其最大凝胶 分数。

有些研究认为 ${ }^{[30,32]}$, 沉积到基板上的液晶弹性 体齐聚物经过 DIW 打印后, 需尽快通过紫外光照固 定液晶基元，以免沉积到基板后液晶基元在余热的 作用下继续运动, 从而趋向于向无序转变, 即液晶 基元由各向异性向各向同性转变, 降低液晶弹性体 


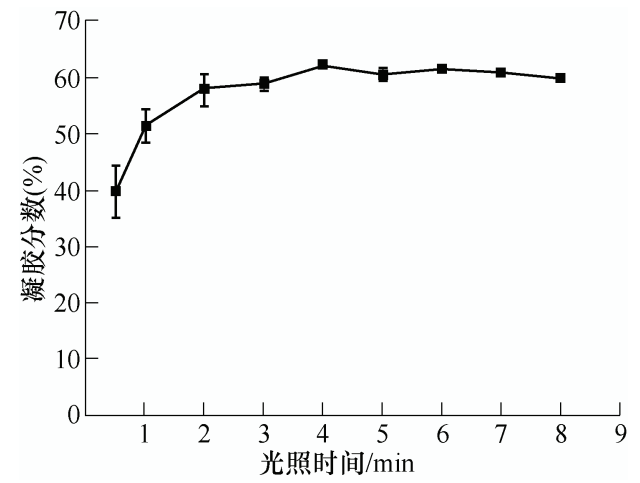

图 11 液晶弹性体凝胶分数与紫外光照时间的关系

的变形率。因此, 在 DIW 打印过程中, 通常需安装 一个紫外灯, 以便在打印的同时进行光照固化。然 而, 这样存在两个问题: (1) 液晶弹性体光照前和光 照后材料性能发生变化, 其黏度和模量等均存在较 大差异，因此光照前的材料和光照后的材料结合性 较差; (2) 由于紫外灯光斑直径通常是固定的, 而打 印单道宽度可能会经常改变, 会存在反复装卸和调 整紫外灯的问题, 或者是光照不均匀带来的材料性 能不均问题。因此, 研究 DIW 打印与紫外光照的间 隔时间对揭示液晶弹性体性能的影响规律, 对设备 和工艺的优化设计具有重要意义。

液晶弹性体变形率与 DIW 打印和光照的间隔 时间的关系如图 12 所示, 从图中可以发现, 间隔 时间对液晶弹性体的变形率在误差范围内可认为 没有影响。这可能是因为环境温度低于 $25{ }^{\circ} \mathrm{C}$, 从针头挤出的材料很快降温, 余热不足以使液晶 基元发生各向同性转变。正因为液晶弹性体变形 率与打印和光照的间隔时间的关系不大, 那么在 DIW 打印的同时无须进行紫外光照, 可以待打印 完一层后再进行光照固化。这样既简化了工艺, 还能优化设备。

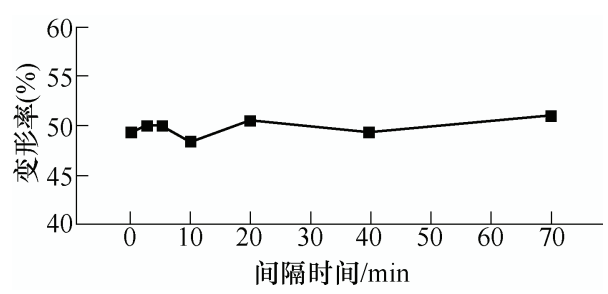

图 12 液晶弹性体变形率与打印和光照的间隔时间的关系

\subsection{DIW 工艺编程设计制备 LCE 器件}

当用两种不同打印速率打印出来的液晶弹性体 结合在一起时, 即由不同打印速率组合成的双层液 晶弹性体, 由于两层的变形率不同, 相互之间会产 生内应力, 使得材料发生弯曲变形。图 13 和图 14 是利用不同打印速率设计的双层液晶弹性体弯曲变 形结果, 较大的打印速率为 $90 \mathrm{~mm} / \mathrm{min}$, 较小的打
印速率为 $30 \mathrm{~mm} / \mathrm{min}$ 。

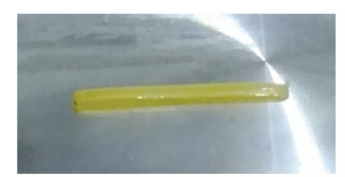

(a) 刚放置热板时 $(0 s)$

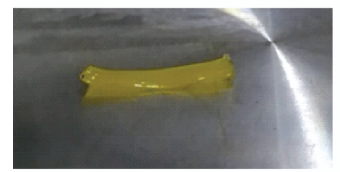

(c) 开始弯曲时(13.3 s)

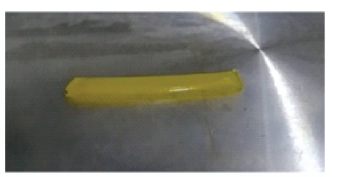

(b) 收缩过程中 $(7.3 \mathrm{~s})$

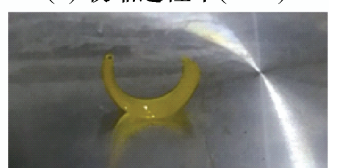

(d) 弯曲到最大程度时(16.6s)
图 13 上层打印速率较大的双层液晶弹性体弯曲变形过程

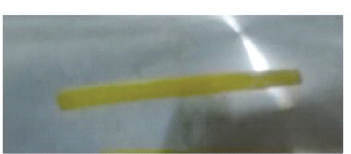

(a) 刚放置上去时(0s)

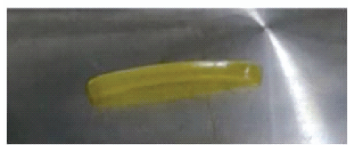

(c) 开始弯曲时(6.3 s)

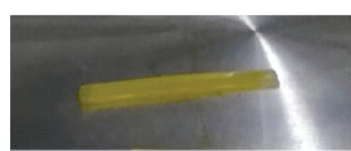

(b) 收缩过程中 $(3.4 \mathrm{~s})$

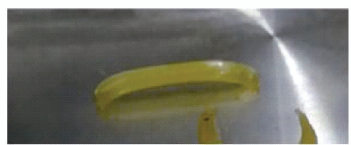

(d) 弯曲到最大程度时(15.5 s)
图 14 下层打印速率较大的双层液晶弹性体弯曲变形过程

如图 13 所示, 把双层液晶弹性体放置在 $150{ }^{\circ} \mathrm{C}$ 的热板上, 其中上层打印速率较大, 刚放 置上去时材料呈二维平面形状紧贴热板平面, 长 度为 $30 \mathrm{~mm}$, 随着放置时间的增加, 液晶弹性体 的温度逐渐增加, 在热的刺激下逐渐变短。当放 置时间达到 $13.3 \mathrm{~s}$ 时材料开始发生弯曲变形, $16.6 \mathrm{~s}$ 时液晶弹性体的变形程度达到最大值。值 得注意的是, $16.6 \mathrm{~s}$ 以后材料会反复进行弯曲-变 直-弯曲过程, 这是因为当液晶弹性体的温度足够 高时将逐渐弯曲到最大值, 弯曲以后液晶弹性体 的温度在空气中迅速下降, 当温度低于其 $T_{\mathrm{NI}}$ 后, 材料将发生各向同性态-液晶态的相变, 上下两层 之间的变形程度差异将消失, 两层之间的应力随 之消失，因此材料又将变长变直。

图 14 所示为下层打印速率较大的双层液晶弹 性体放置在 $150{ }^{\circ} \mathrm{C}$ 热板上的弯曲变形过程, 弯曲方 向与图 13 相反。证明了可以通过打印速率控制液晶 弹性体的变形方向。

前面已经证明了不同打印速率的双层液晶弹性 体可产生弯曲变形。然而, 这种双层液晶弹性体所 产生的弯曲变形是否特定可控弯曲变形有待证实。

图 15 为 DIW 打印的两层液晶弹性体打印速率组合 与弯曲变形曲率间的关系图, 从图中可以发现, 随 着两层之间打印速率差异的增大, 液晶弹性体变形 曲率越大，类似线性方式增长。 


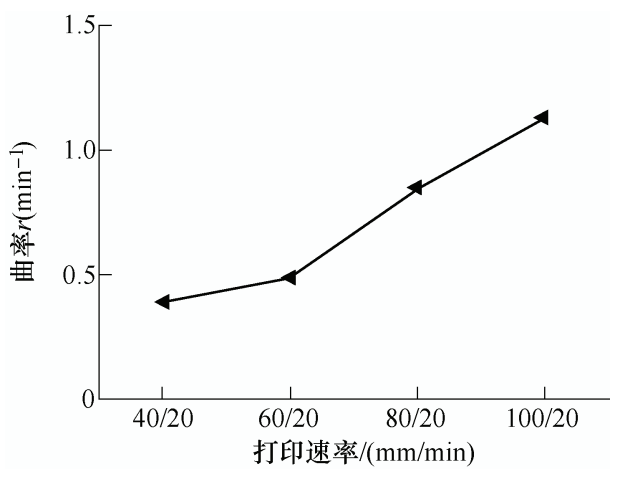

图 15 液晶弹性体弯曲的曲率与打印速率组合之间的关系

在第 2.2 节里已经研究了打印速率与材料变形 率之间的关系, 发现材料变形率与打印速率之间具 有明显的规律性。当变形程度的差异大小不同时, 两 层材料之间的应力大小也不同, 从而使得其弯曲程度 存在差异, 即弯曲的曲率不同。因此可以方便地利用 打印速率编程设计弯曲程度, 液晶弹性体的这一特性 可以很好地被应用于制作抓手或者传感器。

如图 16 所示, 根据上述液晶弹性体的弯曲变形 设计方法, 如果两层的打印速率不同, 高打印速率在 外侧, 低打印速率在内侧, 从而阿拉伯数字 “ 3 ” 可 以通过打印一个英文字母 “L” 后经热激励变形得 到。图 17 是数字 “ 3 ” 在加热前后的实际变形结果。
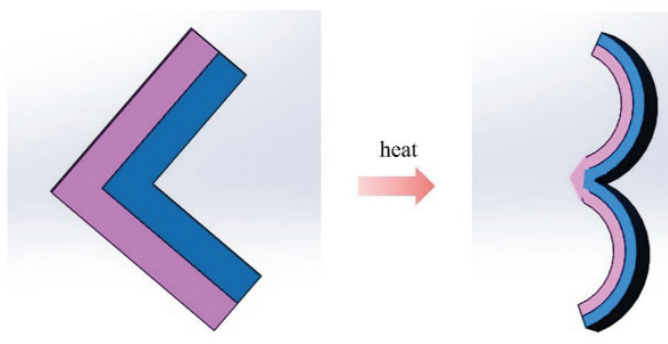

图 16 数字 “ 3 ” 变形原理示意图
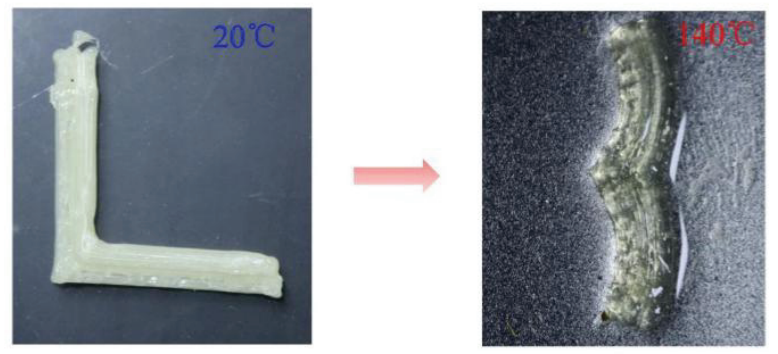

图 17 数字 “ 3 ” 的实际变形效果

实际打印出来的是一个 “ $L$ ” 形英文字母, 其 中内侧是低速率打印出的具有一定宽度的 “ $L ”$, 外侧是高速率打印出的同宽度的 “ $\mathrm{L}$ ”, 由于是同 一种材料, 两种工艺打印出来的材料没有多材料打 印时存在的界面结合问题。加热以后, 在变形程度
差异导致的应力驱动下材料发生弯曲变形, 最后呈 阿拉伯数字 “ 3 ” 状。可以预期的是, 打印件的弯曲 程度可以通过不同打印速率组合设计和同一打印速 率的打印宽度来调整。

\section{3 结论}

（1）利用胺烯迈克尔加成反应原理制备了最大 变形率达 $51.8 \%$ 的液晶弹性体, 温度对材料的黏度 有显著的影响, 打印时需要通过加热来调控材料的 打印性能。

(2) 研制了气压挤出的高温墨水直写 4D 打印 设备, 可加热范围为常温至 $150{ }^{\circ} \mathrm{C}$, 挤出压力大小 为 $0.07 \sim 0.8 \mathrm{MPa}$, 可以实现液晶弹性体的顺利打 印, 并可以对工艺参数进行精准调控。

（3）揭示了直写打印工艺参数对液晶弹性体性 能的影响规律。打印速率可使液晶弹性体变形率在 12\% 51.8\%之间变化。打印速率对沉积到基板上的 线条尺寸也有较大影响。75 110 ${ }^{\circ} \mathrm{C}$ 的打印温度可 以使液晶弹性体的变化率在 $38.3 \% \sim 51 \%$ 之间。紫 外灯功率密度为 $0.8 \mathrm{~mW} / \mathrm{cm}^{2}$ 时, 光照 $4 \mathrm{~min}$ 即可使 材料达到其最大的凝胶分数 $62.2 \%$ 。打印与紫外灯 光照的间隔时间对变形率没有影响, 可以在每层打 印结束后再进行光照固化。

(4) 建立了 DIW 4D 打印速率编程设计对液晶 弹性体变形模式的调控方法。通过不同打印速率的 组合可以设计出弯曲曲率不同的变形模式, 用 $40 / 20 \mathrm{~mm} / \mathrm{min}$ 的打印速率组合成形的双层液晶弹性 体弯曲曲率为 0.39 , 而用 $100 / 20 \mathrm{~mm} / \mathrm{min}$ 的打印速 率组合的双层液晶弹性体弯曲曲率达 1.13 。此外, 利用打印速率成功设计并打印出了具有复杂变形模 式的液晶弹性体, 证明了方法的可行性。

液晶弹性体以其优越独特的性能受到了广泛关 注, 然而在实际应用中仍然存在较大局限, 因此以 下几个方面必然会成为未来的研究重点方向。首先 是材料的制备工艺简化以及性能提升。现阶段液晶 弹性体的制备工艺已经非常成熟，但是其性能仍然 对反应条件较为敏感。而且, 液晶弹性体工作条件 下的模量和黏度等仍然有待提升优化。其次, 液晶弹 性体应用研究逐渐深入, 它在传感器、驱动器、光学 功能材料和吸能材料等方面有着巨大的应用潜力。最 后, 液晶弹性体的成形工艺研究也将是一个重要方 向。近年来得到较大发展的直写打印成形方法在成形 精度和成形速度方面存在其内在局限性, 因此液晶弹 性体的成形方法仍将是人们需要克服的障碍。 


\section{参 考 文 献}

[1] 宋波, 卓林蓉, 温银堂, 等. 4D 打印技术的现状与未 来 $[\mathrm{J}]$. 电加工与模具, $2018(6):$ 1-7.

SONG Bo, ZHUO Linrong, WEN Yintang, et al. The status and future of $4 \mathrm{D}$ printing technology[J]. Electromachining \& Mould, 2018 (6): 1-7.

[2] SABINA W U, NICHOLAS A T, ROSS H V, et al. Liquid crystal elastomers: An introduction and review of emerging technologies[J]. Liquid Crystals Reviews, 2018, 6 (1): 78-107.

[3] SHAHSAVAN H, LI Y, JAKLI A, et al. Smart biomimetic micro/nanostructures based on liquid crystal elastomers and networks[J]. Soft Matter, 2017, 13 (44): 8006-8022.

[4] KULARATNE R S, KIM H, BOOTHBY J M, et al. Liquid crystal elastomer actuators: Synthesis, alignment, and applications[J]. Journal of Polymer Science Part B: Polymer Physics, 2017, 55 (5): 395-411.

[5] WHITE T J, BROER D J. Programmable and adaptive mechanics with liquid crystal polymer networks and elastomers[J]. Nature Material, 2015, 14 (11): 1087-1098.

[6] BROMMEL F, KRAMER D, FINKELMANN H. Preparation of Liquid Crystalline Elastomers. Advanced Polymer Science, 2012, 250: 1-48.

[7] DEY S, AGRA-KOOIJMAN D, REN W, et al. Soft Elasticity in Main Chain Liquid Crystal Elastomers[J]. Crystals, 2013, 3 (2), 363-390.

[8] FINKELMANN H, KIM S T, MUÑOZ A, et al. Tunable mirrorless lasing in cholesteric liquid crystalline elastomers[J]. Advanced Materials, 2001, 13 (14): 1069-1072.

[9] VAN OOSTEN C L, BASTIAANSEN C W M, BROER D J. Printed artificial cilia from liquid-crystal network actuators modularly driven by light[J]. Nature Materials, 2009, $8(8):$ 677-682.

[10] FLEISCHMANN E K, OHM C, SERRA C, et al. Preparation of soft microactuators in a continuous flow synthesis using a liquid-crystalline polymer crosslinker[J]. Macromolecular Chemistry and Physics, 2012, 213 (18): 1871-1878.

[11] PALAGI S, MARK A G, REIGH S Y, et al. Structured light enables biomimetic swimming and versatile locomotion of photoresponsive soft microrobots[J]. Nature Materials, 2016, 15 (6): 647-653.
[12] ROGÓŻ M, ZENG H, XUAN C, et al. Light-driven soft robot mimics caterpillar locomotion in natural scale[J]. Advanced Optical Materials, 2016, 4 (11): 1689-1694.

[13] DESIMONE A, GIDONI P, NOSELLI G. Liquid crystal elastomer strips as soft crawlers[J]. Journal of the Mechanics and Physics of Solids, 2015, 84 () 254-272.

[14] ZAREK M, MANSOUR N, SHAPIRA S, et al. 4D printing of shape memory-based personalized endoluminal medical devices[J]. Macromolecular Rapid Communications, 2017, 38 (2), 1600628.

[15] MORRISON R J, HOLLISTER S J, NIEDNER M F, et al. Mitigation of tracheobronchomalacia with 3D-printed personalized medical devices in pediatric patients[J]. Science Translational Medicine, 2015, 7 (285): $285 \mathrm{ra} 264$.

[16] VILLAR G, GRAHAM A D, BAYLEY H. A Tissue-Like Printed Material[J]. Science, 2013, 340 (6128): 48-52.

[17] WARE $\mathrm{T} \mathrm{H}$, MCCONNEY M E, WIE J J, et al. Voxelated liquid crystal elastomers[J]. Science, 2015, 347 (6225): 982-984.

[18] WARE T H, WHITE T J. Programmed liquid crystal elastomers with tunable actuation strain[J]. Polymer Chemistry, 2015, 6 (26): 4835-4844.

[19] KOMP A, RÜHE J, FINKELMANN H. A versatile preparation route for thin free-standing liquid single crystal elastomers[J]. Macromolecular Rapid Communications, 2005, 26 (10): 813-818.

[20] SÁNCHEZ-FERRER A, FISCHL T, STUBENRAUCH $\mathrm{M}$, et al. Photo-crosslinked side-chain liquid-crystalline elastomers for microsystems[J]. Macromolecular Chemistry and Physics, 2009, 210 (20): 1671-1677.

[21] LEGGE C H, DAVIS F J, MITCHELL G R. Memory effects in liquid crystal elastomers[J]. Journal De Physique II, 1991, 1 (10): 1253-1261.

[22] LI M H, KELLER P, YANG J, et al. An artificial muscle with lamellar structure based on a nematic triblock copolymer[J]. Advanced Materials, 2004, 16 (21): 1922-1925.

[23] KOMP A , FINKELMANN H. A new type of macroscopically oriented smectic-a liquid crystal elastomer[J]. Macromolecular Rapid Communications, 2007, 28 (1): 55-62.

[24] YAKACKI C M, SAED M, NAIR D P, et al. Tailorable and programmable liquid-crystalline elastomers using a two-stage thiol-acrylate reaction[J]. RSC Advances, 
2015, 5 (25): 18997-19001.

[25] KÜPFER J, FINKELMANN H. Nematic liquid single crystal elastomers[J]. Die Makromolekulare Chemie Rapid Communications, 1991, 12 (12): 717-726.

[26] CAMACHO-LOPEZ M , FINKELMANN H , PALFFY-MUHORAY $\mathrm{P}$, et al. Fast liquid-crystal elastomer swims into the $\operatorname{dark}[\mathrm{J}]$. Nature Materials, 2004, 3 (5): 307-310.

[27] YUAN C, ROACH D J, DUNN C K, et al. 3D printed reversible shape changing soft actuators assisted by liquid crystal elastomers[J]. Soft Matter, 2017, 13 (33): 5558-5568.

[28] DE HAAN L T, SÁNCHEZ-SOMOLINOS C , BASTIAANSEN C M W, et al. Engineering of complex order and the macroscopic deformation of liquid crystal polymer networks $[\mathrm{J}]$. Angewandte Chemie - International Edition, 2012, 51 (50): 12469-12472.

[29] DE HAAN L T, GIMENEZ-PINTO V, KONYA A, et al. Accordion-like actuators of multiple 3D patterned liquid crystal polymer films[J]. Advanced Functional Materials, 2014, 24 (9): 1251-1258.
[30] AMBULO C P, BURROUGHS J J, BOOTHBY J M, et al. Four-dimensional printing of liquid crystal elastomers[J]. ACS Applied Materials and Interfaces, 2017, 9 (42): 37332-37339.

[31] LOPEZ-VALDEOLIVAS M, LIU D, BROER D J, et al. 4D printed actuators with soft-robotic functions[J]. Macromolecular Rapid Communications, 2018, 39 (5): 1700710.

[32] KOTIKIAN A, TRUBY R L, BOLEY J W, et al. 3D printing of liquid crystal elastomeric actuators with spatially programed nematic order[J]. Advanced Materials, 2018, 30(10): 5558-5568.

[33] GANTENBEIN S, MASANIA K, WOIGK W, et al. Three-dimensional printing of hierarchical liquid-crystalpolymer structures[J]. Nature, 2018, 561(7722): 226-230.

[34] DEUTSCH M. Orientational order determination in liquid crystals by x-ray diffraction[J]. Physical Review A, 1991, 44 (12): 8264-8270.

作者简介: 汤桂平, 男, 1991 年出生。主要研究方向 4D 打印。 E-mail: m201770907@hust.edu.cn 宋波(通信作者), 男, 博士, 教授, 博士研究生导师。主要研究方向 3D/4D 打印技术, 材料与结构设计。

E-mail: bosong@hust.edu.cn 